\title{
NATURAL USER INTERFACE-BASED CAR INFOTAINMENT CONTROL SYSTEM
}

\author{
Yang-Keun Ahn and Young-Choong Park \\ Korea Electronics Technology Institute \\ 121-835, 8th Floor, \#1599, Sangam-Dong, Mapo-Gu, Seoul \\ Republic of Korea
}

\begin{abstract}
This article proposes a natural user interface (NUI) system for the control of infotainment content in a smart car that recognizes a user's emotions and gaze with a 2D camera, an eye camera. The configuration of the system used to recognize a user's emotions and gaze is introduced. The methods for recognizing a user's emotions and gaze are described. An experiment was performed to evaluate the performance of emotions and gaze recognition, and to demonstrate the user performance of the system proposed in this article.
\end{abstract}

\section{KEYWORDS}

Smart Car, Natural User Interface, Face Recognition, Eye Tracking

\section{INTRODUCTION}

Smart cars (autonomous vehicles) have recently attracted attention as products in the era of the Fourth Industrial Revolution. When the smart car is completely realized, the environment in automobiles will be changed from a driver-centered focus to a passenger-centered one. As a result, smart cars will become a new space for consuming infotainment content. Various methods of handling infotainment content in smart cars have been suggested, and one of them is the natural user interface (NUI) technology.

The NUI technology has previously been applied as a controller for multiple functions. For example, the 'Golf R Touch' system by Volkswagen is used to control the audio volume, sun roof, and windows of vehicles through the recognition of user gestures. Panasonic employs a system that can measure the number of eye blinks of a driver, extract the facial state, recognize the driver's drowsiness, and control the indoor space accordingly.

This article proposes a NUI-based system that allows infotainment contents to be managed in a smart car through the recognition of emotions and gaze.

\section{SYSTEM}

The system proposed in the present study has the configuration shown in [Figure 1], and includes two user interfaces (UI).

The face UI for recognizing a user's emotions includes a 2D camera that receives images of a user's face. The 2D camera used in this study was a Logitech 920C Webcam, which was separated from a user by a distance of less than $60 \mathrm{~cm}$. The front face images were extracted to determine the emotions.

The gaze UI includes a Tobii Eye Tracker 4C, a gaze tracking camera for the tracking the user's gaze, is installed at the lower end of the monitor pointing toward the eyes of a user who is looking at the monitor.

[Figure 2] shows a demonstration of the emotion recognition feature. The user at a distance of about 60 $\mathrm{cm}$ from the 2D camera has made a surprised facial expression. Among different emotions the surprise emotion was recognized at the highest rate of $80 \%$. 


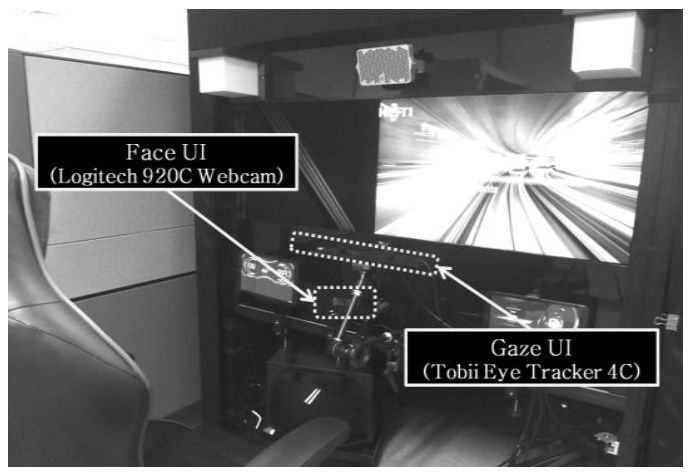

Figure 1. System configuration

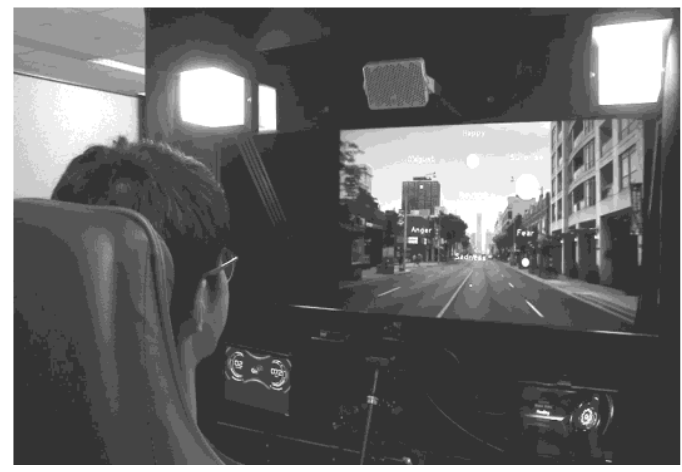

Figure 2. Demonstration of emotion recognition

\section{EMOTION RECOGNITION}

The 2D camera (Logitech 920C Webcam) is located at a position about $60 \mathrm{~cm}$ away from a user so that the front face of the user is shown on the screen. The face is detected from the video input from the 2D camera. After detecting the face, 68 feature points are extracted from the face outline and the area around the eyes, eyebrows, nose, and mouth. The user's emotion is recognized by using the extracted feature points. Seven kinds of emotions may be recognized: Neutral, Happiness, Surprise, Fear, Sadness, Anger, and Disgust. The seven emotions are evaluated by a numerical value ranging from 0 to 100 , and a higher value means that the facial expression is closer to the corresponding emotion.

The emotion recognition software recognizes a user's face. After the face is detected, the user may enter a number from 1 to 9 to connect an ID to the user's face. Later, if the user's face with a predetermined ID is detected, the face ID number is shown on the screen through template matching.

The actions using the facial information, including face detection, feature point extraction, emotion recognition, and face ID recognition, were performed using the functions provided by Verilook SDK (Neurotechnology). [Figure 3] shows the overall flowchart of the emotion recognition software.

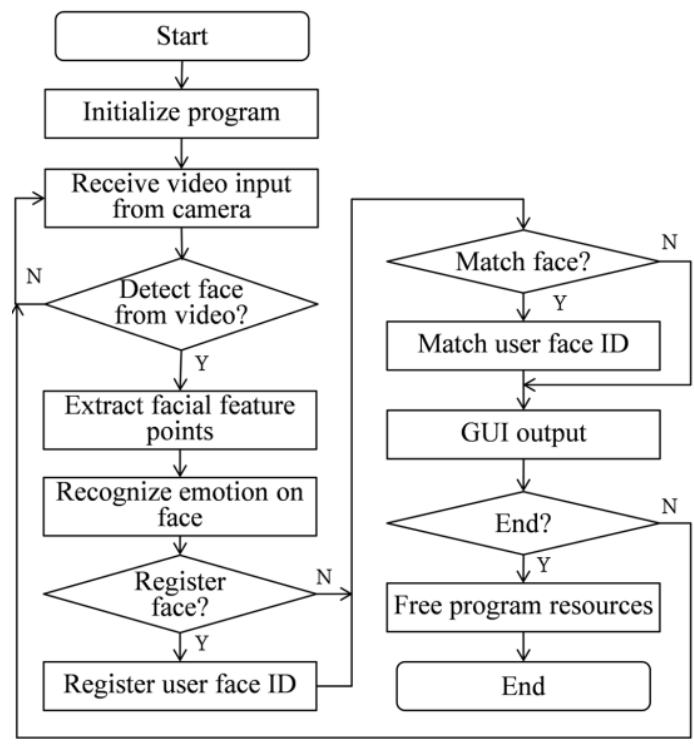

Figure 3. Flowchart of emotion recognition software

[Figure 4] shows the screen in which a user's surprise emotion was recognized. The image on the left shows the user information and the image on the right shows the face information output displayed on the video received from the $2 \mathrm{D}$ image. The face information is of a 28 -year old man whose ID was not 
registered. Among the seven emotions, the recognition values were 95 for surprise, 53 for disgust, and 16 for sadness. The size of the circle in the left image is determined by the numerical values of each emotion. The circle was the largest for the surprise emotion with the highest numerical value.

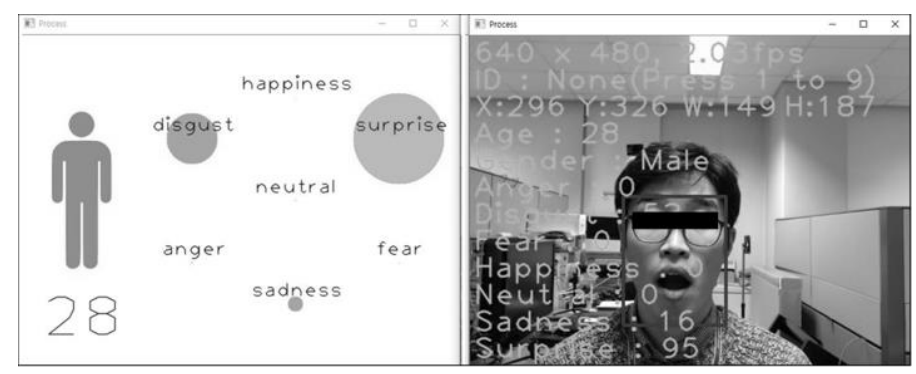

Figure 4. Emotion recognition

\section{GAZE RECOGNITION}

Gaze recognition is performed using Tobii Eye Trakcer 4C, a gaze tracking camera. The position coordinates of the user gaze were obtained by using the function provided by Tobii Eye Tracking Core Software v2.10.0.

The gaze tracking camera was installed at the lower part of the monitor pointing toward the user's eyes. The gaze tracking camera tracks the user's eyes by using the pupil center corneal reflection (PCCR) technology. After the camera is connected, a reflection is produced by a user illuminated with a near-infrared ray, and the camera detects the reflection pattern on the cornea and the pupil. Then, a vector is calculated based on the angle between the cornea and the pupil reflection, and the gaze direction is calculated by combining the vector direction and other geometrical features of the reflection. After that, the point of intersection between the extension line of the user's gaze and the plane of the screen is calculated to obtain the user's gaze coordinates and to output the gaze coordinate information. [Figure 5] shows the overall flowchart of the gaze recognition software.

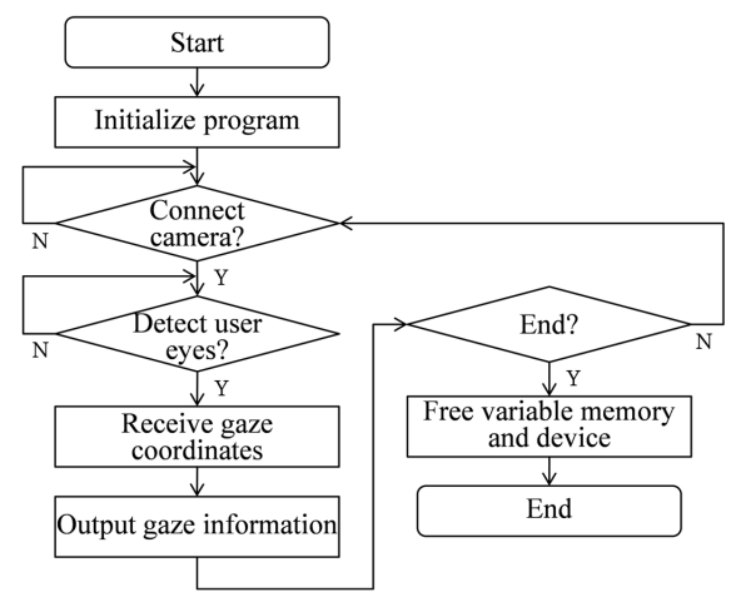

Figure 5. Flowchart of the gaze recognition software

Before using the gaze recognition function, the gaze tracking camera is adjusted to each user by using the calibration function provided by the Tobii Eye Tracker $4 \mathrm{C}$ software. The gaze coordinates values are adjusted to the screen resolution, where the $\mathrm{x}$-coordinate ranges from 0 to 1919 and the $\mathrm{y}$-coordinate ranges from 0 to 1079. When a user's gaze is outside the screen it is specifically processed so that the coordinate values do not exceed the range of the screen resolution.

When the calibration was normally performed for a user, the error of the gaze coordinates was about 1.5 $\mathrm{cm}$ with reference to the screen size $(53 \times 30 \mathrm{~cm})$. 
[Figure 6] shows the screen representing the user gaze recognition results. The user's gaze was directed toward the center of the screen, and the x-coordinate and the y-coordinate of the gaze were 304 and 217, respectively. The point corresponding to the gaze coordinates represents the user's gaze.

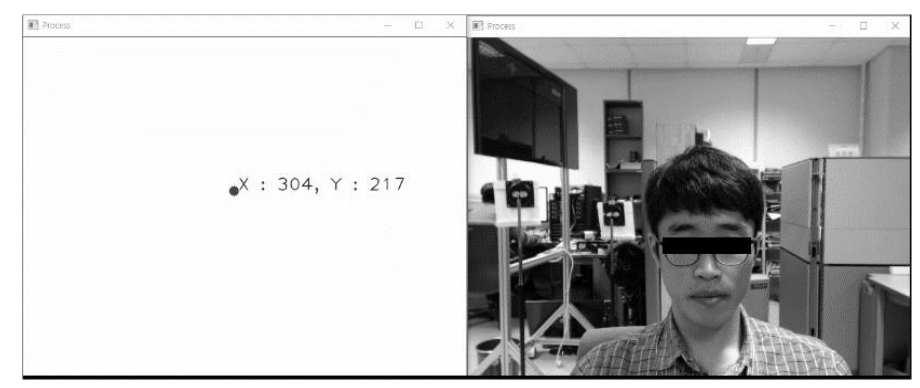

Figure 6. Gaze recognition

\section{EXPERIMENTAL RESULTS}

The performance of the system was tested by connecting the 2D camera and gaze tracking camera to a PC (Intel(R) Core(TM) i7-3770K 3.5 GHz and 8 Gbytes memory).

The emotion and gaze recognition rates were tested to show the performance of the system proposed in this study. Each experiment was performed with five users. The distance between the system and the user was about $60 \mathrm{~cm}$. The 2D camera was installed to view the front face of a user, and the gaze tracking camera was installed at the lower end of the monitor.

The experiment for testing emotion recognition was performed while the five users made the facial expressions for three emotions, of Happiness, Sadness, and Disgust, among the seven kinds of emotions, a hundred times for each emotion. The average of the number of times when the emotion was rightly recognized was calculated. Among the emotions showing a numerical value over 50, if the emotion recognized with the highest numerical value was the same as the user's facial expression, the recognition was considered to be the right recognition.

The emotion recognition experiment showed that the average emotion recognition rate was about $80.13 \%$ [Table 1].

Table 1. Emotion recognition rate

\begin{tabular}{|c|c|c|c|c|}
\hline \multirow{2}{*}{ User } & \multicolumn{3}{|c|}{ Emotion (No. of times) } & \multirow{2}{*}{ Average (\%) } \\
\cline { 2 - 4 } & Happiness & Sadness & Disgust & 81.33 \\
\hline 1 & 76 & 86 & 82 & 81.00 \\
\hline 2 & 93 & 77 & 73 & 77.67 \\
\hline 3 & 85 & 72 & 76 & 81.33 \\
\hline 4 & 68 & 83 & 93 & 79.33 \\
\hline 5 & 74 & 89 & 75 & 80.13 \\
\hline- & - & - & - & \\
\hline
\end{tabular}

Table 2. Gaze recognition rate

\begin{tabular}{|c|c|c|c|c|c|}
\hline \multirow{2}{*}{ User } & \multicolumn{4}{|c|}{ Gaze (No. of times) } & \multirow{2}{*}{ Average (\%) } \\
\hline & Quadrant 1 & Quadrant 2 & Quadrant 3 & Quadrant 4 & \\
\hline 1 & 83 & 88 & 93 & 92 & 89.00 \\
\hline 2 & 86 & 96 & 95 & 93 & 92.50 \\
\hline 3 & 94 & 92 & 91 & 88 & 91.25 \\
\hline 4 & 85 & 84 & 87 & 89 & 86.25 \\
\hline 5 & 93 & 87 & 89 & 97 & 91.50 \\
\hline- & - & - & - & - & 90.10 \\
\hline
\end{tabular}


The gaze was recognized by quartering the screen and letting five users gaze at the centers of the Quadrants 1, 2, 3, and 4, a hundred times for each quadrant. The number of times when the user's gaze point was rightly included in the corresponding quadrant was averaged. The allowed error was about $1.5 \mathrm{~cm}$ from the centers of the quadrants in the screen $(53 \times 30 \mathrm{~cm})$. The average gaze recognition rate found in the experiment was about $90.1 \%$ [Table 2].

\section{CONCLUSION}

The present article proposes a NUI technology-based method of handling infotainment content in a smart car by recognizing a user's emotion and gaze with cameras. This article introduces the system configuration to use the proposed system, and describes the overall flowchart of the software programs for the emotion and gaze recognition UIs, as well as the recognition results. The proposed system does not require a controller with direct contact, such as a mouse and keyboard, but is handled by a user's natural facial expressions and gaze as inputs, and as a result the system may provide convenient and new user experiences.

\section{ACKNOWLEDGEMENT}

This research was supported by Korea Creative Content Agency. (Project No. R2017030009, The development of infotainment contents and interaction for vehicle).

\section{REFERENCES}

Dongmin Shin. et al, 2017. Development of emotion recognition interface using complex EEG/ECG bio-signal for interactive contents. Multimedia Tools and Applications. Vol. 76, No. 9, pp 11449-11470.

Fernández. et al, 2016. Natural user interfaces for human-drone multi-modal interaction. 2016 International Conference on Unmanned Aircraft Systems (ICUAS). Arlington, VA, USA, pp. 838-848.

Sujin Kwak. et al, 2015. Empirical Environment for Smart Car Technologies and Smart Car Talk. Proceeding of Korea Society of Management Information Systems. Republic of Korea, pp. 729-738.

Yejin Shin. et al, 2016. Patent Analysis for Innovative Technology Development of Advanced Driver Assistance Systems (ADAS). Journal of Information Technology and Architecture. Vol. 13, No. 1, pp 147-160. 